\title{
Thermal Contact Conductance at Low Contact Pressures
}

\author{
Fernando H. Milanez* \\ Federal University of Santa Catarina, 88040-900 Florianopolis, SC, Brazil \\ Michael M. Yovanovich ${ }^{\dagger}$ \\ University of Waterloo, Waterloo, Ontario N2L 3G1, Canada \\ and \\ Marcia B. H. Mantelli \\ Federal University of Santa Catarina, 88040-900 Florianopolis, SC, Brazil
}

\begin{abstract}
New correlations are presented for the truncated Gaussian (TG) thermal contact conductance model during first loading. The TG model is also incorporated into existing models for the hysteresis effect of thermal contact conductance and for the plastic contact pressure. The TG models, as well as the existing fully Gaussian models, are compared against new experimental data collected at very low contact pressures. Comparison between the models and the data shows that the fully Gaussian model underpredicts data at low contact pressures, as already extensively reported in the literature. The first-loading TG model predicts the experiments very well over the entire range of the contact pressures tested. The hysteresis effect model proved to be accurate only for contact pressure above $400 \mathrm{kPa}$, in general. The TG model requires a surface roughness parameter, the level of truncation of the probability density function of surface heights, which cannot be obtained accurately from ordinary surface profilometry. The most accurate and straightforward way to estimate this surface geometry parameter is from thermal tests. The levels of truncation of bead-blasted stainless steel 304 and $\mathrm{Ni} 200$ are obtained and presented.
\end{abstract}

\section{Nomenclature}

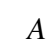

$c_{1}=$ Vickers microhardness correlation coefficient, $\mathrm{Pa}$

$c_{2}=$ Vickers microhardness correlation coefficient

$d_{v}=$ Vickers test indentation diagonal, $\mu \mathrm{m}$

$f \quad=$ truncation function

$H=$ microhardness, $\mathrm{Pa}$

$h_{c}=$ contact conductance, $\mathrm{W} / \mathrm{m}^{2} \cdot{ }^{\circ} \mathrm{C}$

$k=$ thermal conductivity, $\mathrm{W} / \mathrm{m} \cdot{ }^{\circ} \mathrm{C}$

$k_{s}=2 k_{A} k_{B} /\left(k_{A}+k_{B}\right), \mathrm{W} / \mathrm{m} \cdot{ }^{\circ} \mathrm{C}$

$m=$ combined mean absolute slope, $\sqrt{ }\left(m_{A}^{2}+m_{B}^{2}\right), \mathrm{rad}$

$n=$ density of contact spots, $\mathrm{m}^{-2}$

$P \quad=$ contact pressure, $\mathrm{Pa}$

$p=$ interpolation index

$q=$ heat flux, $\mathrm{W} / \mathrm{m}^{2}$

$T=$ temperature, ${ }^{\circ} \mathrm{C}$

$X=$ dummy variable, see Eqs. (16-18)

$Z=$ limit of $X$

$z=$ dimensionless surface height

$z_{\text {trunc }}=$ truncation level of the probability density function of the asperity heights

$\Delta T=$ temperature drop, ${ }^{\circ} \mathrm{C}$

$\lambda=$ dimensionless mean separation gap

$\sigma=$ combined $\mathrm{rms}$ roughness, $\sqrt{ }\left(\sigma_{A}^{2}+\sigma_{B}^{2}\right), \mathrm{m}$

Received 5 May 2003; presented as Paper 2003-3489 at the AIAA 36th Thermophysics Conference, Orlando, FL, 23-26 June 2003; revision received 30 July 2003; accepted for publication 30 July 2003. Copyright (C) 2003 by the authors. Published by the American Institute of Aeronautics and Astronautics, Inc., with permission. Copies of this paper may be made for personal or internal use, on condition that the copier pay the $\$ 10.00$ per-copy fee to the Copyright Clearance Center, Inc., 222 Rosewood Drive, Danvers, MA 01923; include the code 0887-8722/04 \$10.00 in correspondence with the CCC.

*Research Assistant, Satellite Thermal Control Group, Department of Mechanical Engineering; milanez@labsoalr.ufsc.br.

${ }^{\dagger}$ Distinguished Professor Emeritus, Microelectronics Heat Transfer Laboratory, Department of Mechanical Engineering; mmyov@ mhtlab.uwaterloo.ca. Fellow AIAA.

†Professor, Satellite Thermal Control Group, Department of Mechanical Engineering; marcia@labsolar.ufsc.br.

\section{Subscripts}

$\mathrm{A}, \mathrm{B}=$ contacting bodies

$a=$ papparent

$c=$ contact

$v=$ Vickers microhardness test

$r \quad=$ real

$m=$ mean

$\max =$ evaluated at the maximum contact pressure

\section{Introduction}

$\mathbf{H}$ EAT transfer across the interface between two solids has been the subject of study of various researchers in the past decades. Contact heat transfer has many applications in engineering, such as ball bearings, microelectronic chips, and nuclear fuel heat dissipation. Thermal contact resistance is also important in spacecraft applications. The electronic modules necessary for spacecraft missions are attached to the spacecraft structure generally by means of bolted joints or other clamping devices. Thermal contact resistances appear at these interfaces and need to be quantified.

There are several theoretical and experimental studies on the thermal contact conductance in the open literature, especially for the contact between conforming rough surfaces. One of the most accepted theoretical thermal contact conductance models was developed by Cooper, Mikic, and Yovanovich $(\mathrm{CMY})^{1}$ for isotropic surfaces deforming plastically. Other models have been developed based on the CMY model, such as the Mikic ${ }^{2}$ unloading model, among many others. Sridhar and Yovanovich ${ }^{3}$ conducted an extensive review of the most frequently used thermal contact conductance models. They compared the available models against experimental data collected by other researchers and concluded that the CMYbased models are the easiest to use and are very accurate, especially when the surfaces are subjected to relatively high contact pressures. At low contact pressures, the models systematically underestimate experimental data.

Recently, Milanez et al. ${ }^{4}$ proposed an explanation for this unexpected behavior of the thermal contact conductance at low contact pressures. According to these authors, the reason why the models underpredict the experiments at low contact pressures is the truncation of the highest contacting asperities. The CMY-based models, as well as many other theoretical models, assume that the height 
distribution of the asperities that constitute real surfaces is Gaussian. Milanez et al. ${ }^{4}$ showed that this hypothesis is valid only up to some extent: Real surfaces may have Gaussian height distribution up to 4.5 times the rms roughness, $\sigma$, approximately, but they generally do not have asperities above $4.5 \sigma$. In other words, the highest asperities are shorter than predicted by the Gaussian model. Because the highest asperities are shorter than expected, the mean separation gap between contacting surfaces at low contact loads is smaller than predicted. As a consequence, the contact conductance is larger than predicted. As the contact pressure increases, more and more asperities that are not truncated come into contact. The effect of the few truncated asperities then becomes negligible, and the Gaussian model is accurate at higher contact pressures.

Song ${ }^{5}$ and Milanez et al. ${ }^{4}$ proposed the basis for a new theoretical model for thermal contact conductance at low contact pressures: the truncated Gaussian (TG) model. This new model, which is also based on the CMY model, is developed in more detail here. A simple correlation is provided for this model. The TG model is also incorporated here into the model proposed by Mikic ${ }^{2}$ to predict the hysteresis effect of contact conductance. The Song and Yovanovich ${ }^{6}$ model for the plastic contact hardness is also modified according to the TG model. The models are then compared against new thermal contact conductance data carefully collected under very light contact pressures during two loading/unloading cycles.

\section{Review of Thermal Contact Conductance Models}

Real surfaces obtained by the actual machining processes present deviations from their idealized geometry. When real surfaces are analyzed in a microscopic scale, roughness and waviness can be observed. When two bodies are put into contact, they will touch each other only at their highest asperities. These asperities are deformed, generating small contact spots. The real contact area is only a very small fraction of the apparent area. In the remaining portion of the apparent contact area, the two surfaces are separated by small gaps.

The heat transfer through the interface between two solids can take place by three different mechanisms: conduction through the contact spots, radiation through the gap between the solids, and conduction through gas or fluid that may fill the gap. These three mechanisms are generally treated separately, and the thermal conductance of the joint is the summation of the three parts: contact, radiation, and gap conductances. There are numerous theoretical models available in the literature to predict these heat transfer mechanisms. In this work, a vacuum environment is considered, which means that the gap conductance is negligible. Also, small temperature differences and relatively low-temperature levels are considered, meaning that radiation can also be neglected. Therefore, in this work, the thermal conductance of the interface is a function of the heat flow through the contact spots only.

The existing thermal contact conductance models can be classified according to the deformation mode assumed for the contacting asperities: elastic, plastic, or elastoplastic. Under plastic deformation, the asperities are permanently deformed during first loading and do not recover their original shape after the surfaces are pulled apart. Under elastic deformation, as the surfaces are pulled apart the asperities recover the original shape. For the elastoplastic case, some intermediate behavior between fully plastic and fully elastic deformation is observed.

The elastic models predict exactly the same behavior for thermal contact conductance during both ascending and descending levels of contact pressure, that is, there is no hysteresis. On the other hand, the plastic models were developed only for the first loading between the contacting surfaces. During unloading, the contact spots are larger than predicted by the plastic models because of the permanent deformation of the asperities during first loading. As a consequence, the thermal contact conductance in descending pressure levels is greater than during the first loading. This phenomenon is known as hysteresis effect of contact conductance.

The CMY based models will be employed here because a comparative study presented by Sridhar and Yovanovich ${ }^{3}$ showed that these models are easy to manipulate and accurate to predict stain- less steel (SS) 304 and Ni 200 contacts, which are the materials employed in the experimental study developed here.

The CMY based models can be divided into three submodels: thermal, geometrical, and deformation models. The thermal model predicts the thermal contact conductance for a given set of contact parameters, which are the shape, mean size, and number of contact spots. The contact parameters are obtained using a particular deformation model and a geometry model. Cooper et al. ${ }^{1}$ employed the Gaussian model for the surface asperity height distribution and assumed random distribution of asperities over the apparent contact area. In these models, it is also assumed that the surface roughness is isotropic, and therefore, the contact spots are approximately circular in shape.

\section{Thermal Model}

According to the Cooper et al. ${ }^{1}$ thermal model, the thermal contact conductance between conforming isotropic rough surfaces as a function of the contact parameters is given by

$$
h_{c}=\frac{2 k_{s} n a}{\left(1-\sqrt{A_{r} / A_{a}}\right)^{\frac{3}{2}}}
$$

where $n$ is the density of contact spots per unit apparent area, $A_{r} / A_{a}$ is the real-to-apparent contact area ratio, and $k_{s}=2 k_{A} k_{B} /\left(k_{A}+k_{B}\right)$ is the harmonic mean of the thermal conductivities of the two bodies. The contact parameters $a, n$, and $A_{r} / A_{a}$ are obtained from the surface geometry and the deformation models. These models are presented in more detail in the remainder of this section.

\section{Fully Gaussian Geometry Model}

By assuming that the distributions of the surface heights and slopes are independent from each other and follow the Gaussian distribution, as well as assuming that the surfaces undergo plastic deformation during first loading, Cooper et al. ${ }^{1}$ presented an analysis to derive expressions for the contact parameters. Yovanovich ${ }^{7}$ presented the contact parameter expressions for the isotropic plastic model in a more convenient form as follows:

$$
\begin{gathered}
n=\frac{1}{16}\left(\frac{m}{\sigma}\right)^{2} \frac{\exp \left(-\lambda^{2}\right)}{\operatorname{erfc}(\lambda / \sqrt{2})} \\
a=\sqrt{\frac{8}{\pi}} \frac{\sigma}{m} \exp \left(\frac{\lambda^{2}}{2}\right) \operatorname{erfc}\left(\frac{\lambda}{\sqrt{2}}\right) \\
\lambda=\sqrt{2} \operatorname{erfc}^{-1}\left(2 \frac{A_{r}}{A_{a}}\right) \\
\frac{A_{r}}{A_{a}}=\frac{P}{H_{c}}
\end{gathered}
$$

where $\sigma$ and $m$ are the combined rms roughness and mean absolute slope of the surface asperities, respectively, which are geometrical parameters that must be obtained from surface profilometry. The apparent contact pressure is $P$, and the contact plastic hardness is $H_{c}$. Substituting the expressions for the contact parameters [Eqs. (25)] into the thermal model (Eq. 1), one obtains

$$
C_{c} \equiv \frac{h_{c} \sigma}{k_{s} m}=\frac{\sqrt{2}}{4 \sqrt{\pi}} \frac{\exp \left(-\lambda^{2} / 2\right)}{\left(1-\sqrt{P / H_{c}}\right)^{\frac{3}{2}}}
$$

Yovanovich $^{7}$ presented the following simple correlation for Eq. (6):

$$
h_{c} \sigma / k_{s} m=1.25\left(P / H_{c}\right)^{0.95}
$$

\section{TG Geometry Model}

There are numerous works in the literature, such as Milanez et al. $^{4}$ and Song 5 , among many others, showing that the distribution of asperity heights of surfaces obtained by bead blasting, lapping, and grinding are approximately Gaussian. The Gaussian 
geometry model is easy to implement into the CMY-based thermal contact conductance models. Milanez et al. ${ }^{4}$ proposed new expressions for the contact parameters according to the TG model. The TG model assumes that the distribution of surface heights follow the Gaussian distribution up to a defined value $z_{\text {trunc }}$, which is the truncation level. There are no asperities higher than the truncation level, that is, $z \leq z_{\text {trunc }}$. The contact parameters according to the TG model are given by

$$
a=\sqrt{1-\frac{\operatorname{erfc}\left(z_{\text {trunc }} / \sqrt{2}\right)}{\operatorname{erfc}(\lambda / \sqrt{2})}} \frac{2 \sqrt{2}}{\sqrt{\pi}} \frac{\sigma}{m} \exp \left(\frac{\lambda^{2}}{2}\right) \operatorname{erfc}\left(\frac{\lambda}{\sqrt{2}}\right)
$$

where

$$
\lambda=\sqrt{2} \operatorname{erfc}^{-1}\left[2\left(P / H_{c}\right)+\operatorname{erfc}\left(z_{\text {trunc }} / \sqrt{2}\right)\right]
$$

The density of contact spots $n$ and the real-to-apparent area ratio $A_{r} / A_{a}$ are computed using the same expressions as for the fully Gaussian model. As discussed by Milanez et al., ${ }^{4}$ the density of contact spots $n$ must be computed using the same expression as the fully Gaussian model, Eq. (2), because, for a given mean separation gap $\lambda$ smaller than $z_{\text {trunc }}$, the number of asperities higher than $\lambda$ is still the same as if the distribution were Gaussian. This expression would lead to errors only for $\lambda>z_{\text {trunc }}$ because there are no asperities higher than $z_{\text {trunc }}$. Therefore, according to the TG geometry model used here, all of the asperities that were supposed to be higher than $z_{\text {trunc }}$ have a maximum height of $z_{\text {trunc }}$. For the real-to-apparent area ratio $A_{r} / A_{a}$, Eq. (5) is still valid because it was derived based on a force balance between the contacting surfaces and does not depend on the type of surface geometry model employed. When Eqs. (2), (5), (8), and (9) are substituted into the thermal model, Eq. (1), one gets the expression for the thermal contact conductance according to the TG geometry model. Because the final expression is difficult to manipulate, the present authors developed the following novel correlation for the Milanez et al. ${ }^{4}$ TG thermal contact conductance model:

$$
h_{c}=1.25(m / \sigma) k_{s}\left(P / H_{c}\right)^{0.95}[1+1 / f]^{0.9289} \sqrt{1-1 /(1+f)}
$$

where

$$
f=P / H_{c} \sqrt{2 \pi} z_{\text {trunc }} \exp \left(z_{\text {trunc }}^{2} / 2\right)
$$

is a function that takes into account the truncation effect on the thermal contact conductance. In the limiting case where $z_{\text {trunc }} \rightarrow \infty$, that is, the distribution of surface heights is fully Gaussian, $f \rightarrow \infty$ and Eq. (10) gives exactly the same correlation as proposed by Yovanovich $^{7}$ for fully Gaussian distribution of surface heights, presented here as Eq. (7). Therefore, the CMY model is the limiting case of the TG model when the surface height distribution is not truncated. In practical applications, for $f>40$ the difference between the fully Gaussian model, Eq. (7), and the TG model, Eq. (10), is less than $1 \%$. The preceding correlation was obtained by substituting $\operatorname{erfc}(x)$ and $\operatorname{erfc}(x)^{-1}$, appearing in Eqs. (8) and (9), by correlations available by Yovanovich. ${ }^{7}$ The correlation gives a maximum difference of $4 \%$ compared with the exact expression in the range of $10^{-6}<P / H_{c}<10^{-2}$ and $3<z_{\text {trunc }}<4.5$.

\section{Plastic Contact Hardness}

An important parameter appearing in the models presented earlier is the dimensionless plastic contact pressure $P / H_{c}$. Song and Yovanovich ${ }^{6}$ proposed a model to compute this parameter. Assuming a fully Gaussian distribution for the surfaces heights, they developed the following model

$$
\left.\left(P / H_{c}\right)\right|_{\text {Gaussian }}=\left[P / c_{1}(1.62 \sigma / m)^{c_{2}}\right]^{1 /\left(1+0.071 c_{2}\right)}
$$

where $c_{1}$ and $c_{2}$ are the Vickers microhardness correlation coefficients of the softer of the two contacting surfaces. In this expression, $\sigma$ must be in micrometers. The $c_{1}$ and $c_{2}$ coefficients are obtained by performing microhardness tests for several indentation loads (between 10 and $200 \mathrm{~g}$ ) and then correlating the measured Vickers hardness values $H_{v}$ to the respective diagonal of lengths $d_{v}$ of the square impressions left by the indenter in the following form

$$
H_{v}=c_{1}\left(d_{v}\right)^{c_{2}}
$$

As already mentioned, the plastic contact hardness model presented earlier assumes fully Gaussian distribution of surface asperity heights. In the next sections, the present authors propose novel models for the plastic contact hardness and for the hysteresis effect of thermal contact conductance according to the TG geometry model.

\section{Plastic Contact Hardness According to the TG Geometry Model}

Employing the same procedure as Song and Yovanovich ${ }^{6}$, the present authors developed the following correlation for the dimensionless contact pressure $P / H_{c}$, according to the TG model:

$$
P / H_{c}=\left[\left(P /\left.H_{c}\right|_{\text {Gaussian }}\right)^{-p}+\left(P /\left.H_{c}\right|_{\mathrm{TG}}\right)^{-p}\right]^{-(1 / p)}
$$

where

$\left.\left(P / H_{c}\right)\right|_{\mathrm{TG}}$

$=\left\{\left[P / c_{1}(2.178 \sigma / m)^{c_{2}}\right]\left[\operatorname{erfc}\left(z_{\text {trunc }} / \sqrt{2}\right)\right]^{0.4289 c_{2}}\right\}^{1 /\left(1+0.5 c_{2}\right)}$

and $p=3.9+52 \exp \left(10 c_{2}\right)$. In the limiting case where $z_{\text {trunc }} \rightarrow \infty$, Eq. (15) goes to zero and Eq. (14) shows that $P / H_{c}$ is equal to Eq. (12), which was developed for Gaussian surfaces, as already mentioned. In this work, Eq. (14) is used to compute the dimensionless contact pressure $P / H_{c}$, which is an input to the TG thermal contact conductance model, Eqs. (10) and (11).

\section{Hystereis Effect Model According to the TG Geometry Model}

The models presented so far are valid only during the first loading of contact pressure, when the surfaces are deformed plastically. Mikic $^{2}$ developed a model to predict the contact parameters $n, a$, and $A_{r} / A_{a}$ during unloading for surfaces experiencing plastic deformation during first loading. The present authors adapted Mikic's ${ }^{2}$ model, which was developed assuming a fully Gaussian geometry model, according to the TG model.

When the contacting pair is pressed against each other up to a maximum contact pressure $P_{\max }$ and then the contact load is decreased to a new contact pressure $P_{1}$ so that $P_{1}<P_{\max }$, the thermal contact conductance at $P_{1}$ is larger than the value obtained at $P_{1}$ during first loading. This behavior is due to the hysteresis effect of thermal contact conductance, as mentioned before. Mikic ${ }^{2}$ proposed an explanation for this phenomenon as follows: During first loading, the asperities deform plastically up to the maximum contact pressure $P_{\max }$, and then, during subsequent unloading, the asperities recover part of the deformation elastically. However, because the plastic deformation is not completely recovered during unloading, the real contact area during unloading is larger than during first loading for the same contact pressure. Therefore, the contact conductance during unloading is larger than during first loading. If the surfaces are then reloaded, the deformation is elastic and reversible, that is, it is possible to return to the same value of contact conductance at $P_{\max }$ as during the first loading. Mikic ${ }^{2}$ proposed an analytical model for the contact parameters during unloading for surfaces undergoing plastic deformation during first loading. The present authors then adapted the unloading model proposed by Mikic ${ }^{2}$ to incorporate the TG geometry model. The set of equations for the contact parameters during unloading, taking into account for the TG geometry model are given by 


$$
\begin{aligned}
& \frac{P}{H_{\max }}=\left[\frac{4 \pi}{(2 \pi)^{\frac{3}{2}}} \int_{Z}^{\infty}\left(\lambda_{\max }+X^{2}\right) \exp \left[-\frac{\left(\lambda_{\max }+X^{2}\right)^{2}}{2}\right] X^{3}\left(1-\frac{Z}{X}\right)^{\frac{3}{2}} \mathrm{~d} X /\right. \\
& \left.\quad\left(1+\left\{\frac{1}{2} \operatorname{erfc}\left(\frac{z_{\text {trunc }}}{\sqrt{2}}\right) / \frac{4 \pi}{(2 \pi)^{\frac{3}{2}}} \int_{Z}^{\infty}\left(\lambda_{\max }+X^{2}\right) \exp \left[-\frac{\left(\lambda_{\max }+X^{2}\right)^{2}}{2}\right] X^{3}\left(1-\frac{Z}{X}\right) \mathrm{d} X-\frac{1}{2} \operatorname{erfc}\left(\frac{z_{\text {trunc }}}{\sqrt{2}}\right)\right\}\right)\right] \\
& \frac{A_{r}}{A_{a}}=\frac{4 \pi}{(2 \pi)^{\frac{3}{2}}} \int_{Z}^{\infty}\left(\lambda_{\max }+X^{2}\right) \exp \left[-\frac{\left(\lambda_{\max }+X^{2}\right)^{2}}{2}\right] X^{3}\left(1-\frac{Z}{X}\right) \mathrm{d} X-\frac{1}{2} \operatorname{erfc}\left(\frac{z_{\text {trunc }}}{\sqrt{2}}\right) \\
& n=n_{\max }-\frac{1}{4} \int_{0}^{Z} \frac{\exp \left[-\left(\lambda_{\max }+X^{2}\right)^{2}\right]}{\operatorname{erfc}\left[\left(\lambda_{\max }+X^{2}\right) / \sqrt{2}\right]}\left\{\lambda_{\max }-\frac{1}{\sqrt{2 \pi}} \frac{\exp \left[-\left(\lambda_{\max }+X^{2}\right)^{2} / 2\right]}{\operatorname{erfc}\left[\left(\lambda_{\max }+X^{2}\right) / \sqrt{2}\right]} X \mathrm{~d} X\right. \\
& a=\sqrt{\frac{A_{r}}{A_{a}} \frac{1}{n \pi}}
\end{aligned}
$$

where $H_{\max }$ is the plastic hardness, $\lambda_{\max }$ is the mean separation gap, and $n_{\max }$ is the number of contact spots, all evaluated at the maximum contact pressure $P_{\max }$. These parameters are computed using Eqs. (14), (9), and (2), respectively. The model requires an iterative procedure to compute the contact parameters. For a given contact pressure $P<P_{\max }$, Eq. (16) is numerically solved for $Z$. Then $Z$ is substituted into Eqs. (17-19) to compute the contact parameters $A_{r} / A_{a}, n$, and $a$. The contact parameters are finally substituted into Eq. (1) to obtain the contact conductance $h_{c}$. Equations (16-19) can also reproduce the fully Gaussian model by simply setting $z_{\text {trunc }} \rightarrow \infty, z_{\text {trunc }}=5$ in practical applications. In this case, Eqs. (16-19) give exactly the same expressions as the fully Gaussian model proposed by Mikic. ${ }^{2}$

\section{Experimental Study}

The experimental study consisted of measuring the thermal contact conductance between two SS 304 or two Ni 200 specimens under vacuum environment. The test specimens have cylindrical shape, $25 \mathrm{~mm}$ diameter by $45 \mathrm{~mm}$ long, and the contact surfaces are nominally flat. The contact surface of one of the specimens is smooth (lapped), and the contact surface of the other is rough (bead blasted). Three roughness levels of each metal were tested.

\section{Experimental Setup}

The experimental setup is shown in Fig. 1. It consists of a cold plate, a test column, a load cell, an electrical heater, and a loading mechanism. The cold plate is a hollow copper cylinder filled with glycol at a controlled temperature. The test column, consisting of two test specimens and one ARMCO iron flux meter, is placed on the cold plate. The electrical heater is placed on the top of the ARMCO flux meter and can dissipate up to $60 \mathrm{~W}$. Radiation heat losses from the test column are minimized by surrounding the column with a polished aluminum tube.

The load is applied to the test column by means of an articulated arm. The loading arm is connected to a shaft. The extremity of the shaft is external to the vacuum chamber and is connected to a nut as shown in Fig. 1. The nut can be rotated by means of a wrench, applying or releasing load to the test column. Between the nut and the chamber base there is a spring that is designed to absorb the thermal expansion of the testing column, avoiding contact pressure variations as a function of temperature variations of the test column during heating. The contact load is read by means of a calibrated load cell assembled in series with the test column and connected to the data acquisition system.

Six 36-type $\mathrm{T}$ thermocouples are attached to each test specimen and to the ARMCO flux meter. The thermocouples are positioned $5 \mathrm{~mm}$ apart from each other along the longitudinal direction. The thermocouple voltages are read by a data acquisition system controlled by a personal computer. Every $5 \mathrm{~s}$, the computer receives the 18 thermocouple voltages, as well as the load cell signal and the electrical heater voltage. The thermocouple signals are converted into temperature values, and the load cell signal is converted into contact load, using predefined correlations. The computational code uses a least-square method to find the best linear fit for the temperature distribution inside each test specimen and inside the ARMCO flux meter. The heat fluxes of each sample are obtained by multiplying the slope of the temperature distributions by the respective conductivities. The conductivities of the ARMCO iron flux meter and of the SS 304 and Ni 200 test specimens are computed using the following correlations:

$$
\begin{array}{rlrl}
k_{\mathrm{ARMCO}} & =74.6-0.069 T & & 0<T<100^{\circ} \mathrm{C} \\
k_{\mathrm{SS} 304} & =17.7+0.028 T & & 4<T<90^{\circ} \mathrm{C} \\
k_{\mathrm{Ni} 200} & =90.27-0.18 T & 5<T<90^{\circ} \mathrm{C}
\end{array}
$$

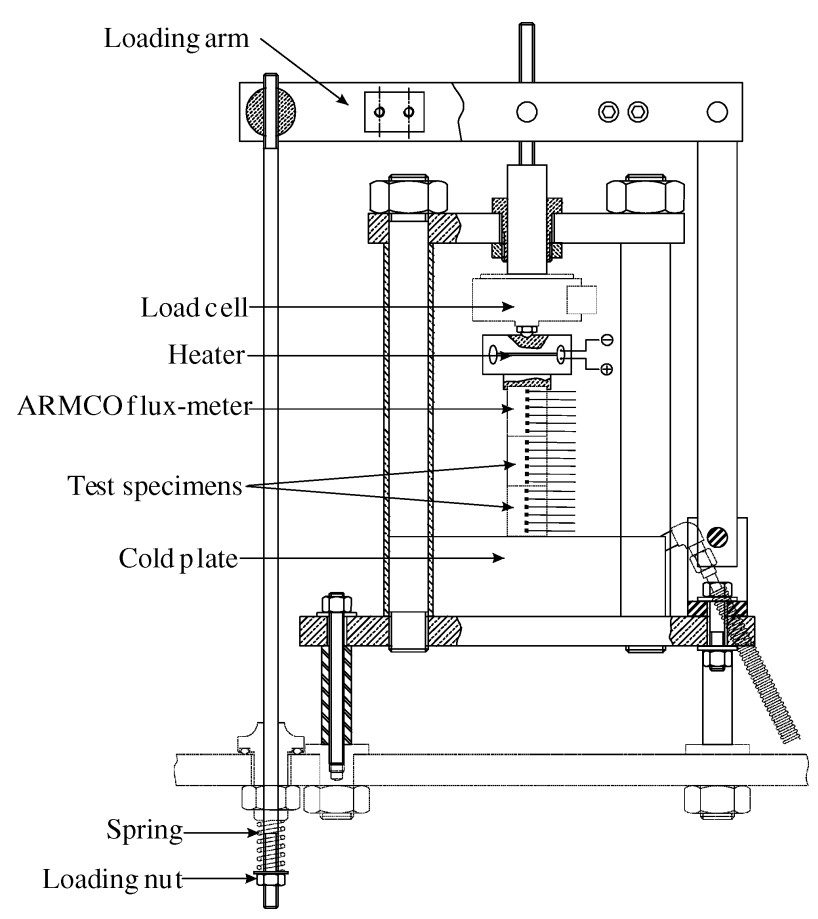

Fig. 1 Experimental setup. 
The correlation for the ARMCO iron was obtained using the tabulated values available in the literature. ${ }^{8}$ The correlations for the SS 304 and Ni 200 were obtained in a previous conductivity test. The conductivity test employed the same experimental setup described here. One SS 304 (or Ni 200) specimen was placed between two ARMCO flux meters. The conductivity of the sample was found by dividing the average heat flux of the two ARMCO flux meters by the slope of the temperature distribution inside the specimen. This conductivity value was assigned to the mean temperature of the specimen. The tests were performed at various mean temperature levels of the specimens. The measured values of conductivity were then correlated to the respective mean temperatures of the specimens, yielding Eqs. (21) and (22).

\section{Test Procedure}

The test pair, consisting of a flat, lapped specimen and a beadblasted surface, was assembled inside the vacuum chamber with no load applied by the loading arm. Therefore, the first contact pressure level (15.8 kPa for SS 304 and $16.2 \mathrm{kPa}$ for $\mathrm{Ni} 200)$ is determined by the dead weights of the upper specimen, flux meter, and electric heater. The chamber was closed and vacuum was drawn using a mechanical pump connected in series with a diffusion pump. The vacuum inside the chamber was $10^{-6}$ torr. The electrical heater was turned on and the system was left for at least $16 \mathrm{~h}$ to achieve steady state.

The thermal contact conductance was computed by means of the following expression:

$$
h_{c}=q / \Delta T
$$

where $q$ is the average of the heat fluxes of the two contacting specimens and $\Delta T$ is the temperature drop at the interface, which is computed by extrapolating the temperature profiles of each contacting specimen to the interface.

This experimental procedure was repeated for each contact pressure level tested. The pressure levels varied from approximately $16-3000 \mathrm{kPa}$ in both ascending and descending levels. Two loading/unloading cycles were measured for each pair. For the lightest contact pressure tested, steady state was achieved after $12 \mathrm{~h}$. As the contact pressure level was increased, the time spent to achieve steady state became smaller. For the maximum pressure tested, approximately $3000 \mathrm{kPa}$, the time required to achieve steady state was less than $2 \mathrm{~h}$. The system was considered to be in steady state when the thermal contact conductance between the specimens did not vary more than $1 \%$ in $1 \mathrm{~h}$. As the contact pressure was increased between each pressure step, the power level of the electrical heater was increased to maintain a reasonable temperature drop between the samples.

\section{Uncertainty Analysis}

The total uncertainty in thermal conductivity estimation using Eqs. (21) and (22) are 5.8 and $8.1 \%$, respectively. The uncertainties in heat flux measurements are $15 \%$ at $16 \mathrm{kPa}$ and $5.7 \%$ at $3000 \mathrm{kPa}$ of contact pressure. The uncertainties in temperature drop measurements are $0.25 \%$ at $16 \mathrm{kPa}$ and $1.25 \%$ at $3000 \mathrm{kPa}$. When the method described by Holman ${ }^{9}$ is used, the uncertainties of the thermal contact conductance measurements for SS 304 are $\pm 15 \%$ at $16 \mathrm{kPa}$ and $\pm 4 \%$ at $3000 \mathrm{kPa}$. For $\mathrm{Ni} 200$, the uncertainties are $\pm 16 \%$ at $16 \mathrm{kPa}$ and $\pm 6 \%$ at $3000 \mathrm{kPa}$.

\section{Experimental Results and Comparison with Theory}

The mechanical and thermal properties and roughness parameters of the test specimens as well as the range of other test parameters are presented in Table 1 . The tests are named here with a letter followed by a number: S1, S2, S3, N1, N2, and N3. The letter refers to the metal, SS $304(\mathrm{~S})$ and Ni $200(\mathrm{~N})$, and the numbers are related to the roughness of the pair tested: The rougher the pair is, the larger the number, that is, $\mathrm{N} 3$ test specimens have total roughness $\sigma$ larger than $\mathrm{N} 2$ test and so on.

The $\sigma, m$, and $\sigma / m$ values presented in Table 1 are the average of the values obtained from the five measurements taken from each

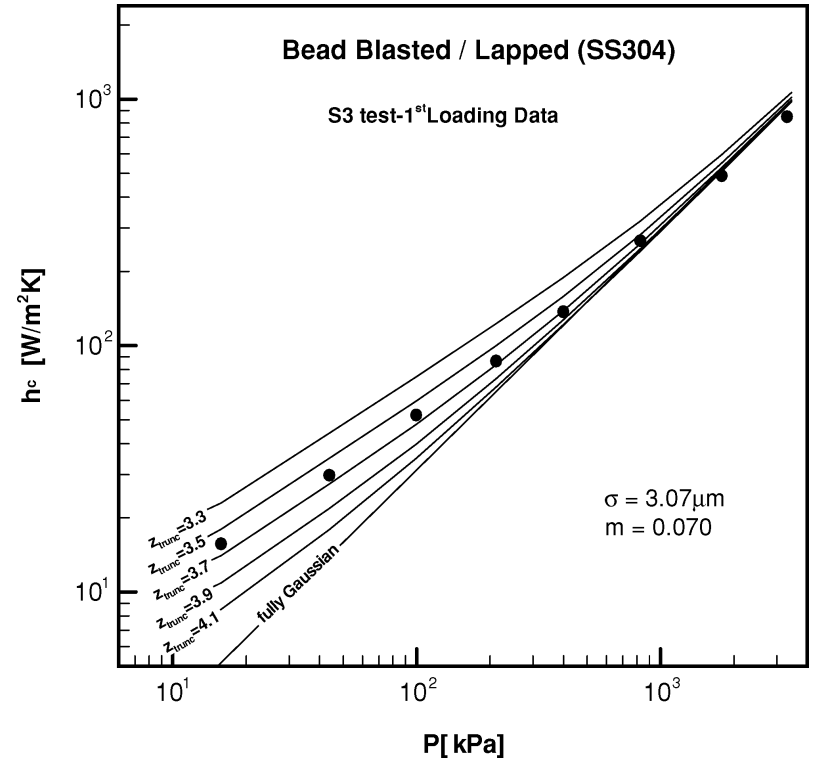

Fig. 2 First-loading data from S3 test and comparison with the TG model for various values of $z_{\text {trunc }}$.

surface by means of a stylus profilometer. The sampling length of the profilometer, that is, the distance between two consecutives asperity height readings, was $1 \mu \mathrm{m}$. The ratio between the standard deviation and the mean value of the five $\sigma$ measurements range between 1 and $10 \%$ for the six test pairs employed in this study. The ratio between the standard deviation and the mean value of the five $m$ measurements range between 2 and $8 \%$ for the six test pairs. The ratio $\sigma / m$ is commonly used as a measure of the roughness level of the surface; it is a better representation of the roughness level than $\sigma$ alone because $\sigma / \mathrm{m}$ appears explicitly in the theoretical models [Eqs. (2), (3), (6), (8), (10), (12) and (15)]. The $c_{1}$ and $c_{2}$ values presented in Table 1 were obtained from five indentations for each load. The average of the 10 diagonal lengths ( 2 diagonals each indentation) and the average of the 5 hardness values for each indentation were used to obtain the correlation coefficients according to Eq. (13). The range of values of the apparent contact pressures $P$, mean interface temperature $T_{m}$, temperature drop $\Delta T$, and heat flux at the interface $q$, as well as the thermal conductivity at the interface $k$, computed as a function of $T_{m}$ according to Eqs. (21) and (22), are also presented in Table 1 .

The values of $z_{\text {trunc }}$ presented in Table 1 were obtained by fitting the correlation of the TG thermal contact conductance model for plastic deformation during the first loading, Eqs. (10) and (11), to the experimental data points. Figure 2 shows this procedure. The first-loading data is plotted in a log-log graph along with a family of curves of the TG model generated with different values of $z_{\text {trunc }}$. For the case presented in Fig. 2 (test S3), the TG model with a value of $z_{\text {trunc }}=3.5$ gives a line that is parallel to the data points; therefore, $z_{\text {trunc }}=3.5$ is adopted as the truncation level in this case. The data points do not have to lie exactly over the best line. Instead, the best line is the one which is parallel to the data points. This procedure avoids that errors of roughness, conductivity, and hardness measurements, which could displace the theoretical curves up or down in the graph, affect the choice of the best theoretical curve. As discussed extensively by Milanez et al. ${ }^{4}$ and addressed later, this is believed to be the only accurate method to estimate $z_{\text {trunc }}$.

Figures 3-5 show $h_{c}$ against $P$ for tests S1-S3. Figures 6-8 present the results for tests N1-N3. The experimental data, as well as the theoretical curves for first loading and unloading models are presented. For first loading, the CMY plastic model with the fully Gaussian geometry model [Eq. (7)] is presented as a dashed line, and the CMY plastic model with the TG geometry model is presented as a continuous line [Eqs. (10)]. For unloading, the Mikic ${ }^{2}$ fully Gaussian model [Eqs. (1), (16), and (17) with $z_{\text {trunc }} \rightarrow \infty$ ] is presented as a dotted line, and the Mikic ${ }^{2}$ model adapted according 
Table 1 Mechanical, thermal, geometrical, and other test parameters

\begin{tabular}{lcccccc}
\hline \hline & \multicolumn{5}{c}{ Test } \\
\cline { 2 - 7 } Parameter & $\mathrm{S} 1$ & $\mathrm{~S} 2$ & $\mathrm{~S} 3$ & $\mathrm{~N} 1$ & $\mathrm{~N} 2$ & $\mathrm{~N} 3$ \\
\hline$\sigma / m, \mu \mathrm{m}$ & 17.7 & 26.2 & 40.0 & 23.0 & 27.0 & 48.0 \\
$\sigma$ (bead blasted), $\mu \mathrm{m}$ & 0.7 & 1.3 & 3.9 & 1.7 & 3.0 & 4.2 \\
$m$ (bead blasted) & 0.036 & 0.047 & 0.098 & 0.074 & 0.11 & 0.086 \\
$\sigma$ (lapped), $\mu \mathrm{m}$ & 0.12 & 0.12 & 0.12 & 0.10 & 0.10 & 0.10 \\
$m$ (lapped) & 0.022 & 0.022 & 0.022 & 0.017 & 0.017 & 0.017 \\
Total flatness deviation, $\mu \mathrm{m}$ & 0.9 & 0.8 & 0.7 & 0.6 & 0.6 & 0.6 \\
$c_{1}, \mathrm{GPa}$ & 10.67 & 10.67 & 10.67 & 4.33 & 4.33 & 4.33 \\
$c_{2}$ & -0.37 & -0.37 & -0.37 & -0.079 & -0.079 & -0.079 \\
$z_{\text {trunc }}$ & 4.1 & 3.8 & 3.5 & 4.2 & 4.2 & 3.9 \\
$k, \mathrm{~W} / \mathrm{m} \cdot \mathrm{K}$ & $18.4-19.5$ & $18.3-19.4$ & $18.2-19.2$ & $84.2-87.3$ & $84.8-87.3$ & $85.8-87.0$ \\
$P, \mathrm{kPa}$ & $15.8-2768$ & $15.8-2720$ & $15.8-3450$ & $16.2-1970$ & $16.2-1780$ & $16.2-2080$ \\
$H_{c}, \mathrm{GPa}$ & $6.2-3.8$ & $6.6-3.3$ & $6.5-2.7$ & $3.7-3.4$ & $3.6-3.4$ & $3.6-3.2$ \\
$T_{m},{ }^{\circ} \mathrm{C}$ & $23-65$ & $20-63$ & $19-54$ & $16-33$ & $16-30$ & $17.5-24$ \\
$\Delta T,{ }^{\circ} \mathrm{C}$ & $5.5-48$ & $7-85$ & $9-77$ & $9-21$ & $9-19$ & $9-19$ \\
$q, \mathrm{~W} / \mathrm{m}^{2}$ & $290-19,000$ & $365-12,000$ & $280-7980$ & $1200-35,500$ & $1200-41,200$ & $280-7980$ \\
\hline
\end{tabular}

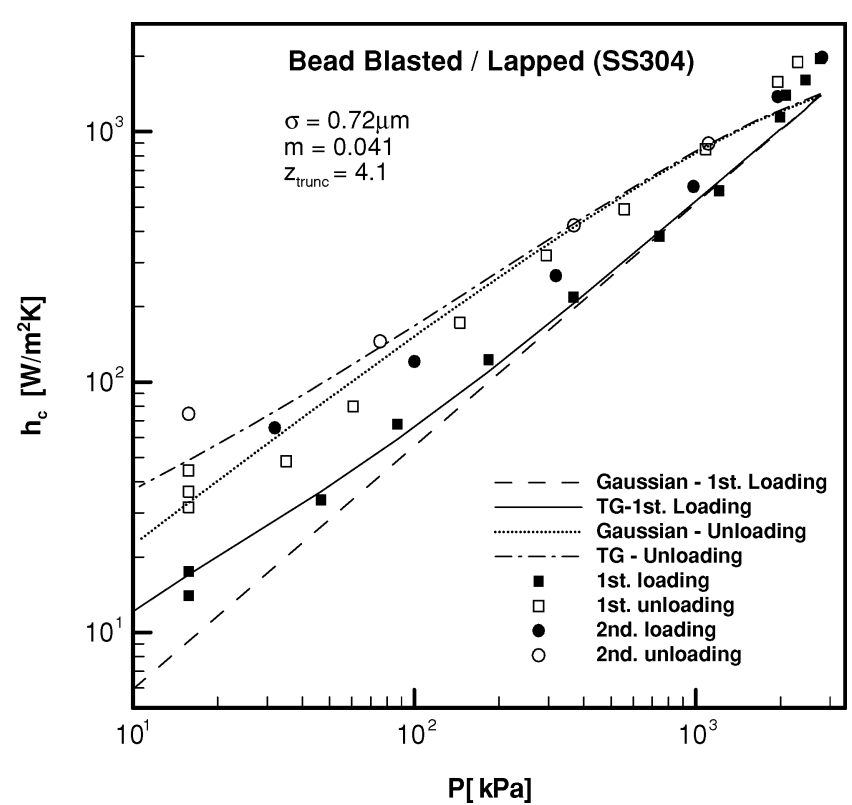

Fig. 3 Results from test S1.

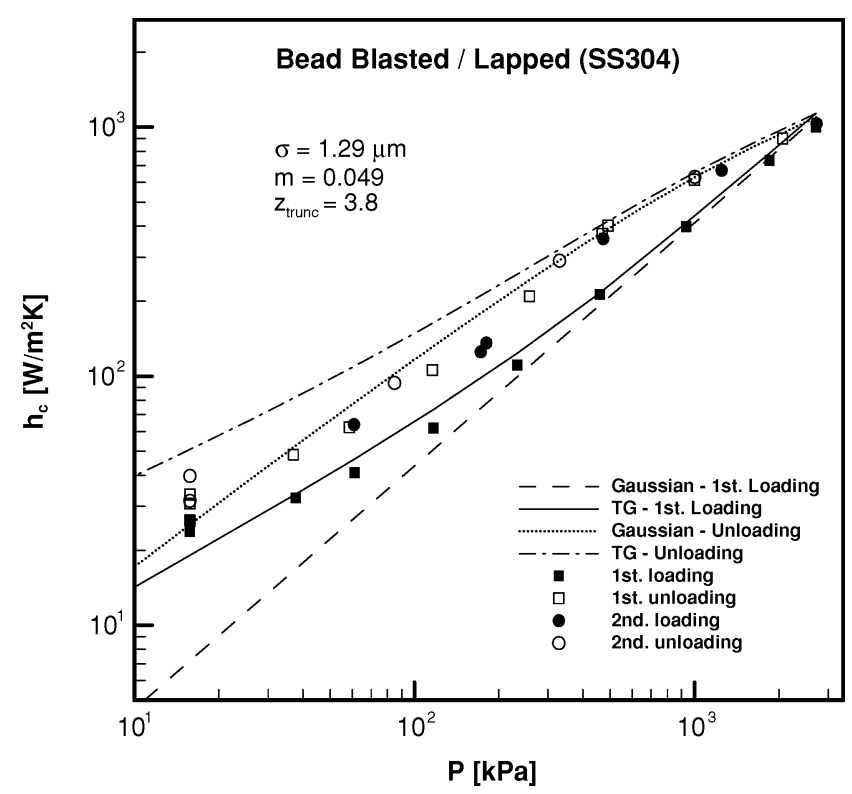

Fig. 4 Results from test S2.

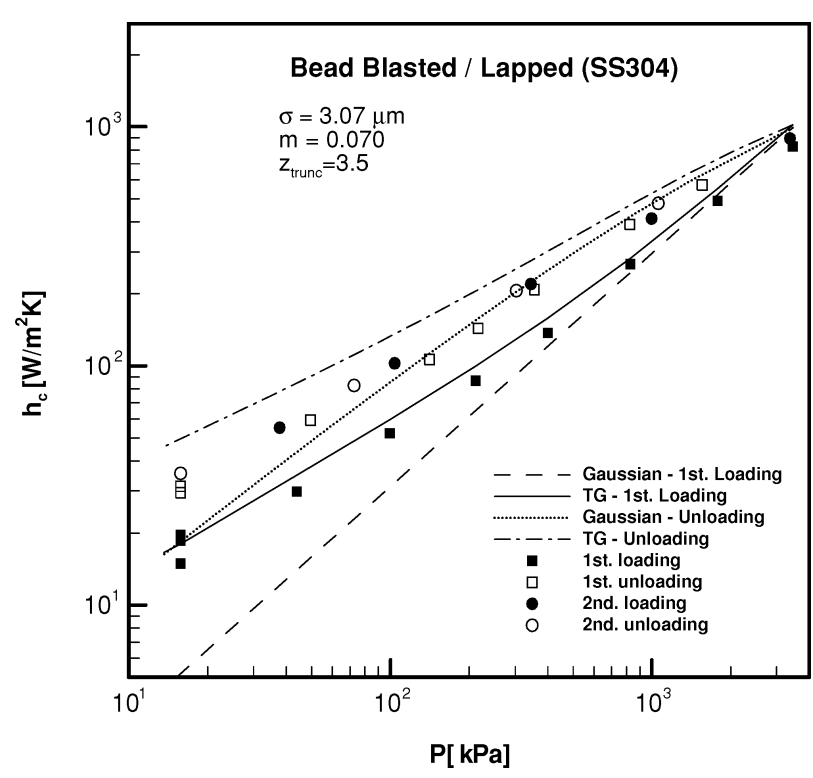

Fig. 5 Results from test $\mathrm{S3}$.

to the TG geometry model [Eqs. (1), (16) and (17) with a finite value of $\left.z_{\text {trunc }}\right]$ is presented as a dash-dotted line.

In general, the results are practically the same for both metals and all roughness levels tested. The first-unloading data points lie above the first-loading data points, which characterizes the hysteresis effect of thermal contact conductance, that is, an indication that plastic deformation took place during the first loading. In general, the hysteresis effect is negligible during the first-unloading/secondloading/second-unloading process, indicating the appearance of elastic deformation. These observations are in agreement with the theory described by Mikic ${ }^{2}$.

The fully Gaussian model underpredicts the first-loading data at light loads, as already observed by Sridhar and Yovanovich, ${ }^{3}$ among others. As the contact pressure increases, the model tends to the measured values. On the other hand, the TG first-loading model with apropriate values of $z_{\text {trunc }}$ predicts the data points over the entire range very well. Table 2 gives the improvement in the agreement between theory and experiments when one uses the TG model instead of the fully Gaussian geometry model. Table 2 shows the rms differences between the measured and the predicted values of thermal contact conductance during first loading according to both the fully Gaussian and the TG models. As one can see, the differences for the TG model are between 5.4 and $17.5 \%$, whereas the differences for the fully Gaussian model are between 21.1 and $48.4 \%$. 
Table 2 Differences between the first-loading data and the models

\begin{tabular}{lcr}
\hline \hline & \multicolumn{2}{c}{ RMS differences [\%] } \\
\cline { 2 - 3 } Test & Gaussian & TG \\
\hline S1 & 25.3 & 16.1 \\
S2 & 44.1 & 17.5 \\
S3 & 48.4 & 15.6 \\
N1 & 21.1 & 8.2 \\
N2 & 25.5 & 5.4 \\
N3 & 41.2 & 8.4 \\
\hline \hline
\end{tabular}

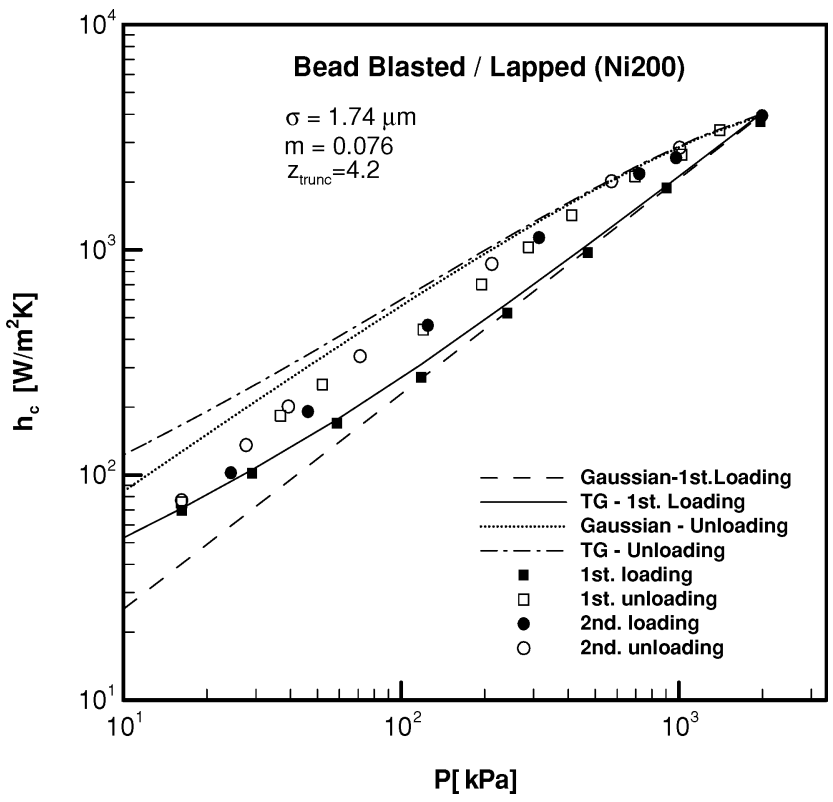

Fig. 6 Results from test N1.

Both unloading models (fully Gaussian and TG) predicted the experimental data fairly well for contact pressure larger than $300 \mathrm{kPa}$. For $P<300 \mathrm{kPa}$, the fully Gaussian unloading model predicted the data better than the TG unloading model, although the TG unloading model predicted the trend of data points better than the fully Gaussian unloading model.

\section{Truncation Levels of Bead Blasted Surfaces}

As seen in Table 1, the $z_{\text {trunc }}$ values that make the TG model better fit the SS 304 data are 4.1,3.8, and 3.5 for $\sigma / m$ values of 17.7, 26.2, and 40.0, respectively. For Ni 200, the $z_{\text {trunc }}$ values are $4.2,4.2$, and 3.9 for $\sigma / m$ values of 23.0, 27.0, and 48.0, respectively. In general, the $z_{\text {trunc }}$ values decrease with the roughness level $\sigma / \mathrm{m}$. The values of $z_{\text {trunc }}$ for Ni 200 are larger than the values for the SS 304 tests for the same roughness level, indicating that the bead-blasting process is able to generate high asperities more likely for $\mathrm{Ni} 200$ than for SS 304.

The values of $z_{\text {trunc }}$ presented in Table 1 can be used as a reference for the truncation levels of bead-blasted SS 304 and Ni 200 surfaces.They can also be used to estimate the truncation levels of bead-blasted SS 304 and Ni 200 for values of $\sigma / m$ other than those tested here by means of linear interpolation. However, it would be very useful if one could measure $z_{\text {trunc }}$ directly from surface profilometry, using the same equipment employed to measure the other roughenss parameters $\sigma$ and $m$. It would be especially helpful to measure $z_{\text {trunc }}$ for other metals and other surface machining processes, because the values presented here are valid only for Ni 200 and SS 304 prepared by bead blasting. Unfortunately, it seems likely that is not the case, as discussed extensively by Milanez et al. ${ }^{4}$ and will be briefly reviewed here.

The stylus profilometer is the most common equipment employed for surface roughness measurements. It consists basically of a sharp

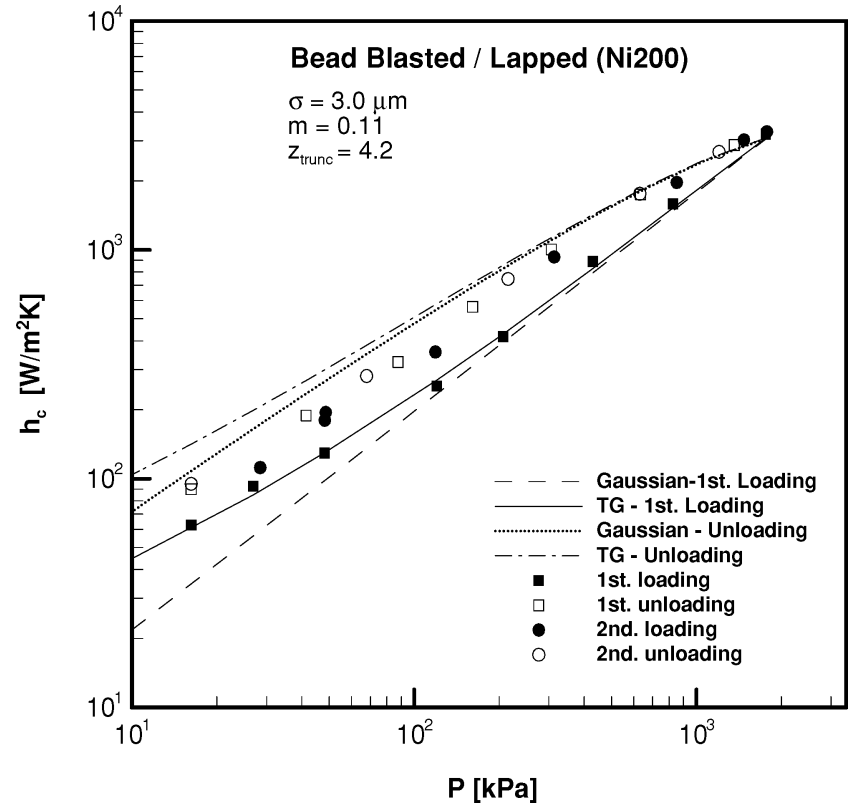

Fig. 7 Results from test N2.

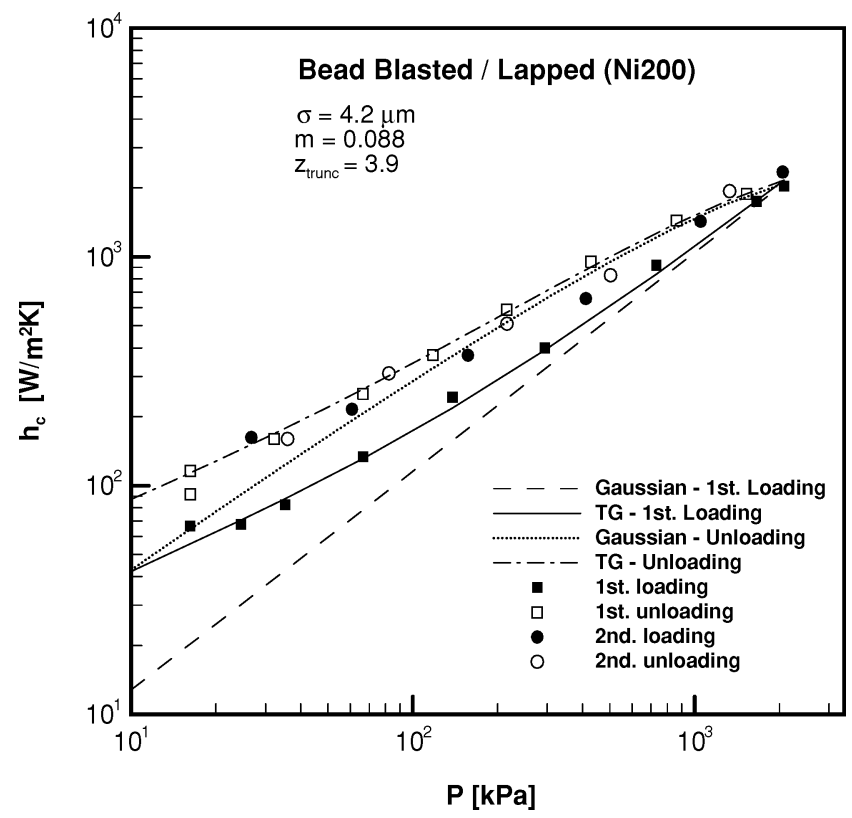

Fig. 8 Results from test N3.

diamond stylus, which is dragged over the surface. As the stylus passes over the asperities that constitute the surface, it moves up and down, and the vertical displacements are measured. At the end of the process, the equipment is able to measure a profile of the surface. The surface height measurements are then converted into roughness parameters such as $\sigma$ and $m$.

Most of the commercially available stylus profilometers measure another surface roughness parameter, generally called $R_{p}$, which is the height of the highest asperity of the profile. The objective now is to compare the $z_{\text {trunc }}$ values available from thermal tests with $R_{p}$ measurements to see whether $R_{p}$ is a good estimation of $z_{\text {trunc }}$ or not. Because $z_{\text {trunc }}$ is the truncation level normalized by the rms roughness $\sigma$, then $R_{p}$ must be divided by $\sigma$ also. Figure 9 presents $136 R_{p} / \sigma$ measurements collected from 24 bead-blasted SS 304 surfaces generated at several roughness levels. As one can see, the points are distributed over a very wide area. In general, for the same roughness level, the measured values of $R_{p} / \sigma$ are spread between 2.5 and 4.5. Rupert ${ }^{10}$ conducted a similar study on surfaces prepared by turning, lapping, and grinding and made similar 


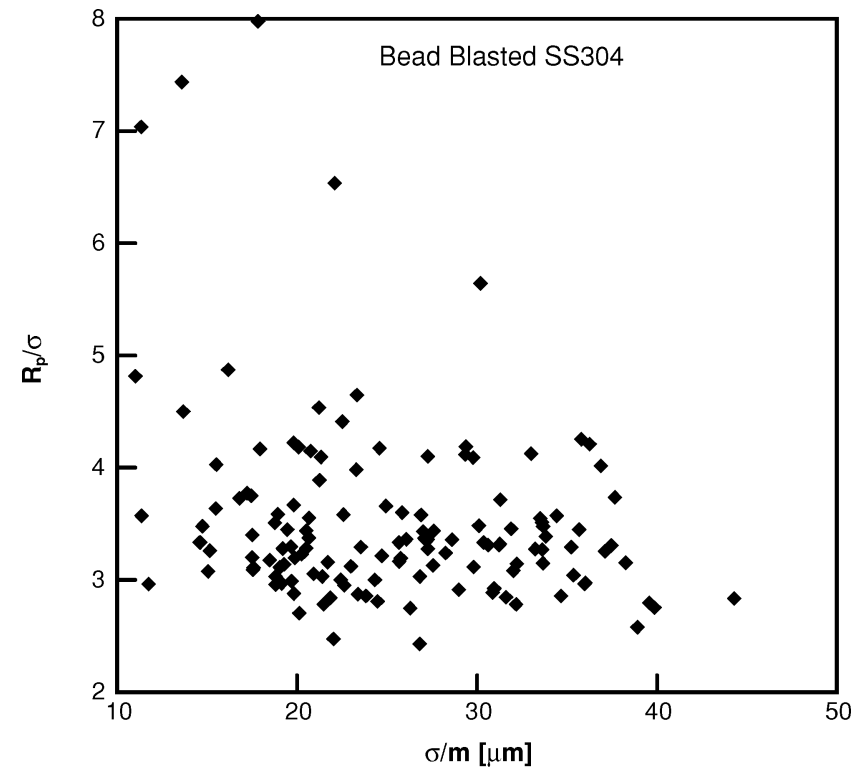

Fig. $9 R_{p} / \sigma$ vs $\sigma / \mathrm{m}$ for bead-blasted SS 304 surfaces.

observations. As shown in Fig. 2, the TG model is very sensitive to the value of $z_{\text {trunc. }}$. At the lightest contact pressure presented in Fig. $2(15.8 \mathrm{kPa})$, for a variation of $z_{\text {trunc }}$ from 4.1 to 3.3 , the model varies from approximately 8 to $20 \mathrm{~W} / \mathrm{m}^{2} \mathrm{~K}$, that is, a $150 \%$ difference. Given the large variation of $R_{p} / \sigma$ values, it is difficult to say which one is the best estimation of $z_{\text {trunc }}$. Therefore, one can conclude that $R_{p} / \sigma$ values obtained from ordinary profilometry are not accurate to estimate $z_{\text {trunc }}$. One must instead rely on the values of $z_{\text {trunc }}$ obtained by fitting the TG model to thermal data to predict accurately the effect of surface roughness truncation on contact conductance. The values obtained in this work for beadblasted SS 304 and Ni 200 can be used to estimate $z_{\text {trunc }}$ for these metals provided the surfaces are prepared by bead blasting. For other metals and other surface preparation methods, new tests are needed.

\section{Summary}

This work presents new correlations for the TG thermal contact conductance model proposed by Milanez et al. ${ }^{4}$ for first loading. Adaptations to the Mikic ${ }^{2}$ unloading model and to the Song and Yovanovich ${ }^{6}$ plastic contact pressure model according to the truncated Gaussian geometry model are also presented here. The models are compared against new thermal contact conductance data collected at very low contact pressures. The comparison between the models and the data show that the first-loading, fully Gaussian, model underpredicts data at low contact pressures, as already extensively reported in the literature. The first-loading TG model predicts the experiments very well over the entire range of the contact pres- sures tested. The Mikic ${ }^{2}$ unloading model proved to be accurate only for contact pressure above $400 \mathrm{kPa}$, in general.

The TG model requires an extra surface roughness parameter, the level of truncation of the probability density function of surface heights $z_{\text {trunc }}$. Unfortunately, this surface roughness parameter cannot be accurately determined from ordinary surface profilometry. The most accurate and straightforward way to estimate $z_{\text {trunc }}$ is from thermal tests. The procedure to measure $z_{\text {trunc }}$ consists of measuring thermal contact conductance for several contact pressure levels in the low contact pressure range (less than $700 \mathrm{kPa}$ ) and then comparing the data with the TG model for several values of $z_{\text {trunc }}$. The value of $z_{\text {trunc }}$ that leads to the best fit between the TG model and the data is the truncation level of the surface height distribution. In this work, truncation levels for bead-blasted SS 304 and Ni 200 for various roughness levels are measured and presented. These values can be used as a reference to predict the truncation levels of beadblasted SS 304 and Ni 200. New tests are needed for other metals and other surface machining processes.

\section{Acknowledgments}

The first and the third authors acknowledge the Brazilian Federal Agency for Postgraduate Education for providing the scholarship and the Brazilian Space Agency for supporting this project. The second author acknowledges the financial support of the Natural Sciences and Engineering Research Council of Canada.

\section{References}

${ }^{1}$ Cooper, M., Mikic, B. B., and Yovanovich, M. M., "Thermal Contact Conductance," Journal of Heat and Mass Transfer, Vol. 12, 1969, pp. 279-300.

${ }^{2}$ Mikic, B. B., "Analytical Studies of Contact of Nominally Flat Surfaces: Effect of Previous Loading," Journal of Lubrication Technology, Oct. 1971, pp. 451-456.

${ }^{3}$ Sridhar, M. R., and Yovanovich, M. M., "Review of Elastic and Plastic Contact Conductance Models: Comparison with Experiment," Journal of Thermophysics and Heat Transfer, Vol. 8, No. 4, 1994, pp. 633-640.

${ }^{4}$ Milanez, F. H., Yovanovich, M. M., and Culham, J. R., "Effect of Surface Asperity Truncation on Thermal Contact Conductance," IEEE Transactions on Components and Packaging Technologies, Vol. 26, No. 1, 2003, pp. 48-54; also VIII Itherm, 29 May-1 June 2002.

${ }^{5}$ Song, S., "Analytical and Experimental Study of Heat Transfer through Gas Layers of Contact Interfaces," Ph.D. Dissertation, Dept. of Mechanical Engineering, Univ. of Waterloo, Waterloo, ON, Canada, Nov. 1988.

${ }^{6}$ Song, S., and Yovanovich, M. M., "Relative Contact Pressure: Dependence on Surface Roughness and Vickers Microhardness," Journal of Thermophysics and Heat Transfer, Vol. 2, No. 4, 1988, pp. 633-640.

${ }^{7}$ Yovanovich, M. M., "Thermal Contact Correlations," Spacecraft Radiative Heat Transfer and Temperature Control, edited by T. E. Horton, Progress in Astronautics and Aeronautics, Vol. 83, AIAA, New York, 1981, pp. 83-95.

${ }^{8}$ Journal of Physical and Chemical Reference Data, Vol. 3, Supplement No. 1, 1974.

${ }^{9}$ Holman, J. P., Experimental Methods for Engineers, 6th ed., McGrawHill, Singapore, 1994, pp. 49-103.

${ }^{10}$ Rupert, M. P., "Confusion in Measuring Surface Roughness," Engineering, 23 Oct. 1959, pp. 393-395. 